\title{
Condición física funcional en adultos mayores institucionalizados
}

\author{
Functional physical condition in institutionalized elderly adults \\ Cindy Lorena Benavides-Rodríguez ${ }^{*}$ orcid.org/0000-0003-0905-1122 \\ José Alexander García-García' ${ }^{1}$ orcid.org/0000-0002-3598-0884 \\ Jairo Alejandro Fernández² orcid.org/0000-0002-6731-2645
}

1. Programa LEFRD. Corporación Universitaria CENDA. Bogotá, Colombia

2. Programa LEFRD. Universidad Pedagógica Nacional. Bogotá, Colombia

\section{Resumen}

Introducción: La cuantificación de la condición física funcional (CFF) en el adulto mayor, es un indicador importante para definir el estado de salud, el nivel de dependencia y la calidad de vida en esta población. Objetivo: Determinar la CFF en un grupo de adultos mayores que residen en hogares geriátricos de la ciudad de Bogotá. Materiales y métodos: Las variables de composición corporal fueron estimadas por antropometría y la condición física funcional valorada con la batería Senior Fitness Test. Resultados: Fueron evaluados 253 adultos mayores $(42,6 \%$ hombres y 57,3\% mujeres), edad $(75,5 \pm 9,6)$ e IMC $(24,9 \pm 3,6)$. Condición física funcional: levantarse y sentarse $(10,9 \pm 3,6$ hombres y 10,6 $\pm 3,5$ mujeres), flexión de brazo $(13,3 \pm 3,3$ hombres y 11,7 $\pm 3,8$ mujeres $)$, flexión de tronco $(-11,4 \pm 8,2$ hombres y $-1,1 \pm 4,8$ mujeres), juntar las manos $(-16,5 \pm 10,7$ hombres y $-7,3 \pm 6,3$ mujeres), capacidad aeróbica (77,4 $\pm 13,6$ hombres y $63,9 \pm 14,2$ mujeres), agilidad y equilibrio $(7,5 \pm 2,3$ hombres y 7,6 $\pm 2,4$ mujeres). Conclusiones: La situación de institucionalización impacta negativamente la condición física funcional del adulto mayor en los componentes de fuerza, agilidad y equilibrio, aumentando el riesgo de caídas y discapacidad física.

Palabras clave: Adulto mayor; condición física funcional; institucionalizados. (Fuente: DeCS, Bireme).

\begin{abstract}
Introduction: Quantification of the functional physical condition (FPC) in the elderly population is an important indicator to define their state of health, level of dependence, and quality of life. Objective: To determine FPC in a group of elderly people residing at nursing homes in the city of Bogotá (Colombia). Materials and methods: Body composition variables were estimated by anthropometry. The functional physical condition was assessed by the Rikli and Jones Senior Fitness Test battery. Results: 253 older adults (42.6\% men and 57.3\% women), their age (75.5 \pm 9.6$)$, and BMI $(24.9 \pm 3.6)$ were evaluated. Functional physical condition values were: getting up and sitting down (10.9 \pm 3.6 for men and 10.6 \pm 3.5 for women), arm flexion (13.3 \pm 3.3 men and $11.7 \pm 3.8$ women), trunk flexion ( $-11.4 \pm 8.2$ men and $-1.1 \pm 4.8$ women), joining hands ( $-16.5 \pm 10.7$ men and $-7.3 \pm 6.3$ women), aerobic capacity (77.4 \pm 13.6 men and $63.9 \pm 14.2$ women), and agility and balance ( $7.5 \pm 2.3$ men and $7.6 \pm 2.4$ women). Conclusions: Institutionalization of the elderly has a negative impact on their functional physical condition. In particular, it affects their strength, agility and balance, which increases the risk of falls and physical disability.
\end{abstract}

Keywords: Older adult; functional physical condition; institutionalized. (Source: DeCS, Bireme).

\footnotetext{
*Autor de correspondencia

Cindy Lorena Benavides Rodríguez

e-mail: cindy.benavides@cenda.edu.co
} 


\section{Introducción}

El proceso de transición demográfica indica que el envejecimiento en la población colombiana, tiene tasas de crecimiento superiores al de la población mundial. Entre 1985 y el año 2013, la población total de 60 años o más aumentó en 1.672.344, con un ritmo de crecimiento promedio del $3,18 \%$ anual en ese periodo. Para el 2015, el incremento de la población mayor fue del $3,51 \%$ y se tiene proyectado para el 2020 , un aumento del 3,76\%(1). Este crecimiento poblacional es más acentuado en ciudades como: Bogotá, Valle, Atlántico, Quindío, que presentan niveles relativamente altos de desarrollo social y transiciones demográficas más avanzadas(2).

El envejecimiento es un proceso progresivo, caracterizado por el deterioro gradual acumulativo y generalizado de las funciones fisiológicas, que puede explicarse por factores genéticos, múltiples morbilidades, y a su vez factores no genéticos especialmente relacionados con la nutrición, el estilo de vida y la actividad física(3); generando, un desgaste muscular y una alteración estructural del sistema nervioso, que limita a los sujetos en su movilidad, presentando una fuerte incidencia en el deterioro del equilibrio y la marcha(4,5). Así mismo, se origina una pérdida gradual de la capacidad funcional, debido a una disminución de la densidad mineral ósea, la fuerza muscular y la calidad muscular, predisponiendo a los adultos mayores a un incremento en el riesgo de discapacidad(6,7); situación, que se evidencia en el aumento de las caídas, reflejando una condición dependencia y movilidad que requiere de cuidados de enfermería o atención hospitalaria(8). Considerando lo anterior, aparecen patologías como la dinapenia debido a las deficiencias funcionales del sistema neuromuscular humano que aunado a la pérdida de fuerza y potencia muscular, impactan de manera directa la condición física funcional en el adulto mayor ${ }^{(9)}$.

Es entonces, que la disminución de la fuerza se da de manera paralela a la pérdida de masa muscular; después de los 20 años y hasta los 70 , se produce una reducción entre el 30 y 40\% de ésta. La pérdida de masa muscular, ocurre principalmente a través del deterioro de unidades motoras y atrofia de las fibras musculares supervivientes; dicha, consunción de fibras musculares está asociada con la muerte de las motos neuronas (MN) en la columna vertebral(10). Por ende, el detrimento de estos dos componentes impacta la distribución de la composición corporal; condiciones que aumentan la prevalencia de caídas, que afectan al 32\% de los adultos con edades entre 65 a 74 años y a $51 \%$ con edad superior de 85 años(11). Por consiguiente, el promedio de disminución en las moto neuronas en adultos mayores de 60 años, es de aproximadamente el 50\%. Asociado a la atrofia muscular, se observa una reducción del $18 \%$ en la sección trasversal del musculo y un 25\% menos de fibras musculares en los adultos mayores en comparación con los adultos jóvenes(12).

Con respecto, a los cambios que ocurren durante el proceso de envejecimiento, la condición física funcional (CFF), se convierte en un elemento fundamental a estudiar, alterar alguno de sus componentes (fuerza muscular, flexibilidad, equilibrio, agilidad y capacidad aeróbica) conduce a la disminución de la capacidad funcional del adulto mayor; entendiendo la CFF, como el conjunto de cualidades físicas necesarias para la realización de las tareas de la vida diaria de una forma segura e independiente sin una fatiga excesiva(13). En este sentido, en los últimos años se ha identificado el mantenimiento de las cualidades físicas (por ejemplo, fuerza, resistencia, potencia agilidad, y el equilibrio), como un factor clave en la preservación de la movilidad y la independencia que permite a los adultos mayores realizar las actividades cotidianas como subir escaleras, levantar y transportar objetos, entrar y salir de la silla de un vehículo, caminar alrededor de las tiendas, edificios o parques(14). Estos niveles de funcionamiento físico son aspectos estrechamente relacionados con el concepto de "envejecimiento exitoso" $(15,16)$.

Con base en lo expuesto, se puede establecer por medio de estudios epidemiológicos, que una baja CFF, se convierte en un predictor significativo de eventos adversos de salud tales como discapacidad(17), hospitalización y mortalidad entre adultos mayores ${ }^{(18)}$, producidos por el pobre funcionamiento muscular que disminuyen la capcidad funcional, aumentando así el número de caídas y mortalidad en esta población(19,20). Es de vital importancia considerar que la CFF, se mejora a partir de la ayuda de la práctica habitual de la Actividad Física y una alimentación adecuada, retrasando el envejecimiento e incrementando la esperanza de vida(21). El propósito del presente estudio fue determinar la CFF en un grupo de adultos mayores que residen en hogares geriátricos de la ciudad de Bogotá. 


\section{Materiales y métodos}

Se trata de un estudio observacional de diseño transversal y de corte descriptivo, que evaluó la condición física funcional en el adulto mayor institucionalizado de la ciudad de Bogotá. La muestra se conformó por 253 adultos mayores institucionalizados en 12 hogares geriátricos de la ciudad Bogotá, que fueron seleccionados a través de la técnica de muestreo no probabilístico por conveniencia. El 42,7\% fueron hombres y mujeres $57,3 \%$, quienes participaron de manera voluntaria tras conocer el propósito del estudio y cumplir con los criterios de inclusión: ser mayor de 60 años, firmar el consentimiento informado y residir permanentemente en una institución de adultos mayores. Los criterios de exclusión estuvieron asociados a la presencia de dolores severos en extremidades, enfermedades respiratorias, insuficiencia cardíaca, episodios de vértigo $\mathrm{y}$ contraindicaciones médicas para esfuerzos físicos.

\section{Evaluación de variables}

La medición de las variables de estudio se realizó en un único día, iniciando por la valoración antropométrica y posteriormente, la valoración de la condición física funcional como se evidencia a continuación:

Variables antropométricas: Se evaluó: la masa corporal total utilizando una báscula electrónica (tanita BF-559, con 0,50 g. de precisión), la estatura con un estadiómetro móvil (SECA-217 rango20 - 205 cm y de $1 \mathrm{~mm}$ de precisión). El IMC se calculó a través de la ecuación teórica IMC= peso $(\mathrm{kg}) /$ Talla $(\mathrm{m})^{2}$.

Condición Física Funcional: Se valoró a través de la batería, Senior Fitness Test (SFT) propuesta por Rikli \& Jones (2001), adoptando el siguiente orden en la realización de las pruebas físicas:

1) Sentarse y levantarse de una silla en $\mathbf{3 0}$ segundos (repeticiones): número de stands completos en 30 seg con los brazos cruzados sobre el pecho.

2) Flexión de codo en $\mathbf{3 0}$ segundos (repeticiones): número de flexiones de bíceps en 30 seg sosteniendo el peso de la mano (mujeres $5 \mathrm{lb}$; hombres $8 \mathrm{lb}$ ).

3) Capacidad aeróbica en 2 minutos (repeticiones): número de pasos completados en $2 \mathrm{~min}$, elevando cada rodilla hasta el punto medio entre la rótula y la cresta ilíaca (la puntuación es el número de veces la rodilla derecha alcanza el objetivo).

4) Flexibilidad de miembros inferiores y superiores (cm): desde la posición sentada al frente de la silla, con la pierna extendida y las manos extendidas hacia los dedos de los pies, número de $\mathrm{cm}(+\mathrm{o}-)$ desde los dedos extendidos hasta la punta del dedo del pie.

5) Juntar las manos (cm): con una mano por encima del hombro y la otra en medio de la espalda, número de $\mathrm{cm}$ entre dedos medios extendidos (+ o -).

6) Agilidad y equilibrio (seg): número de segundos necesarios para levantarse de la posición sentada, caminar 8 pies, girar y volver a la posición sentada en chai.

Los valores obtenidos en el presente estudio, fueron comparados con los valores de referencia propuestos en la batería mencionada.

\section{Análisis estadístico}

Para el análisis estadístico se utilizó el sofware SPSSStatistical Package for Social Sciences versión 23.0 (SPSS Inc., Chicago, IL, USA). Respecto a la verificación de normalidad en la muestra se utilizó la prueba Kolmogorov-Smirnov. Se realizó un análisis descriptivo univariado según el nivel de medición de las variables, de manera que para las variables cuantitativas (peso, talla IMC, medidas por la SFT, entre otras) se calculó la media y la desviación estándar; mientras que para las variables cualitativas (género, grupos etarios, categorías del IMC) se obtuvo la distribución porcentual de frecuencias.

\section{Consideraciones éticas}

El estudio fue diseñado siguiendo las normas deontológicas reconocidas por la Declaración de Helsinki y la Resolución 008430 de 1993 del Ministerio de Salud de Colombia y aprobado por el Comité de Ética de la Universidad Pedagógica Nacional (UPN). A todos los participantes se les explicó los detalles del estudio y firmaron el consentimiento informado.

\section{Resultados}

La muestra estuvo conformada por 253 adultos, 108 $(42,7 \%)$ hombres y $145(57,3 \%)$ mujeres, entre los 60 y 94 años de edad, distribuidos en cinco grupos etarios de la siguiente forma: 60-64 $(n=41), 65-69$ $(n=42), 70-74 \quad(n=37), 75-79(n=42), 80-84(n=36)$, 
85-89 (n=32) y 90-94 (n=23) y según género como se observa en la Figura 1.

Las características de composición corporal por sexo y grupo etario se presentan en la Tabla 1. En relación al estado nutricional, la población del estudio se clasificó siguiendo la propuesta de la Sociedad Española de Geriatría y Gerontología.

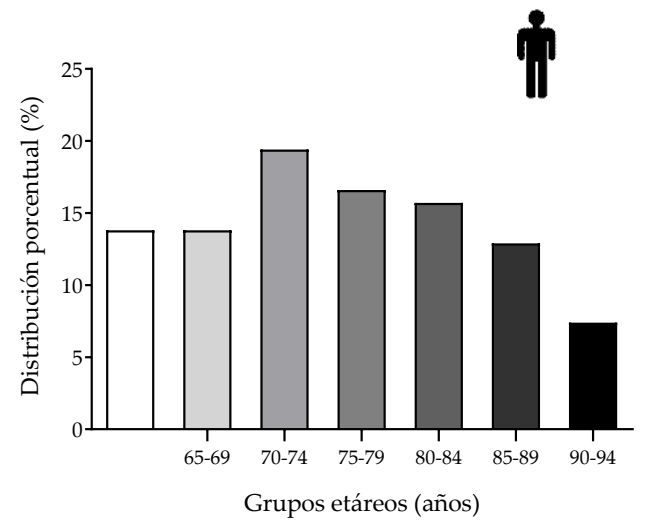

Figura 1. Distribución de la muestra por sexo y rango de edad

Tabla 1. Características de la composición corporal

\begin{tabular}{|c|c|c|c|c|c|c|c|}
\hline Variables & $\begin{array}{c}60-64 \\
n=41\end{array}$ & $\begin{array}{c}65-69 \\
n=42\end{array}$ & $\begin{array}{l}\begin{array}{c}70-74 \\
n=37\end{array} \\
\end{array}$ & $\begin{array}{l}75-79 \\
n=42\end{array}$ & $\begin{array}{c}80-84 \\
n=36\end{array}$ & $\begin{array}{c}85-89 \\
n=32\end{array}$ & $\begin{array}{l}90-94 \\
n=23\end{array}$ \\
\hline \multicolumn{8}{|l|}{ Peso (kg) } \\
\hline Hombres & $63,6 \pm 10,6$ & $61,7 \pm 10,5$ & $61,1 \pm 7,8$ & $64,2 \pm 10,5$ & $61,4 \pm 9,1$ & $62,7 \pm 9,9$ & $53,1 \pm 7,7$ \\
\hline Mujeres & $58,2 \pm 6,8$ & $59,9 \pm 7,9$ & $51,2 \pm 10,4$ & $55,1 \pm 9,8$ & $51,8 \pm 10,2$ & $55,8 \pm 12,6$ & $51,7 \pm 7,6$ \\
\hline \multicolumn{8}{|l|}{ Talla (cm) } \\
\hline Hombres & $158 \pm 0,6$ & $160 \pm 0,1$ & $159 \pm 0,7$ & $163 \pm 0,7$ & $156 \pm 0,8$ & $158 \pm 0,8$ & $151 \pm 0,8$ \\
\hline Mujeres & $151 \pm 0,5$ & $153 \pm 0,5$ & $143 \pm 0,5$ & $147 \pm 0,6$ & $145 \pm 0,7$ & $146 \pm 0,9$ & $144 \pm 0,6$ \\
\hline \multicolumn{8}{|l|}{ Clasificación nutricional } \\
\hline $\mathrm{IMC}\left(\mathrm{kg} / \mathrm{m}^{2}\right)$ & $25,5 \pm 3,3$ & $24,8 \pm 2,9$ & $24,4 \pm 3,7$ & $24,8 \pm 3,7$ & $24,8 \pm 3,6$ & $25,5 \pm 4,4$ & $24,2 \pm 2,9$ \\
\hline Desnutrición Moderada & $2,40 \%$ & $0,00 \%$ & $2,70 \%$ & $2,40 \%$ & $0,00 \%$ & $6,30 \%$ & $0 \%$ \\
\hline Bajo Peso & $12,20 \%$ & $16,70 \%$ & $24,30 \%$ & $21,40 \%$ & $27,80 \%$ & $15,60 \%$ & $17,40 \%$ \\
\hline Peso Normal & $29,30 \%$ & $28,60 \%$ & $40,50 \%$ & $31,00 \%$ & $30,60 \%$ & $28,10 \%$ & $34,80 \%$ \\
\hline Sobrepeso & $46,30 \%$ & $50 \%$ & $21,60 \%$ & $35,70 \%$ & $33,30 \%$ & $31,30 \%$ & $47,80 \%$ \\
\hline Obesidad I & $9,80 \%$ & $4,80 \%$ & $10,80 \%$ & $9,50 \%$ & $8,30 \%$ & $15,60 \%$ & $0 \%$ \\
\hline Obesidad II & $0 \%$ & $0 \%$ & $0 \%$ & $0 \%$ & $0 \%$ & $3,10 \%$ & $0 \%$ \\
\hline
\end{tabular}

Tabla 2. Promedios y deviaciones estándar de la condición física funcional

\begin{tabular}{|c|c|c|c|c|c|c|c|c|c|}
\hline Variables & Sexo & $\begin{array}{c}60-64 \\
n=41\end{array}$ & $\begin{array}{c}65-69 \\
n=42\end{array}$ & $\begin{array}{l}70-74 \\
n=37\end{array}$ & $\begin{array}{l}75-79 \\
n=42\end{array}$ & $\begin{array}{c}80-84 \\
n=36\end{array}$ & $\begin{array}{c}85-89 \\
n=32\end{array}$ & $\begin{array}{l}90-94 \\
n=23\end{array}$ & $p$ \\
\hline Levantarse y & Hombres & $15,1 \pm 1,5$ & $14,5 \pm 1,5$ & $13,4 \pm 0,8$ & $9,8 \pm 1,7$ & $8,2 \pm 0,7$ & $7,1 \pm 1,0$ & $4,8 \pm 0,8$ & $<0,01^{* *}$ \\
\hline sentarse (rep) & Mujeres & $13,9 \pm 9,7$ & $12,9 \pm 9,4$ & $12,3 \pm 1,7$ & $11,7 \pm 1,2$ & $8,7 \pm 1,2$ & $6,7 \pm 1,0$ & $4,0 \pm 1,0$ & $<0,01^{* *}$ \\
\hline Flexión de codo & Hombres & $17,6 \pm 1,3$ & $16,3 \pm 1,8$ & $14,8 \pm 1,0$ & $13,5 \pm 0,7$ & $11,5 \pm 1,0$ & $9,2 \pm 1,0$ & $6,7 \pm 1,2$ & $<0,01^{* *}$ \\
\hline (rep) & Mujeres & $14,6 \pm 1,6$ & $15,2 \pm 1,9$ & $13,4 \pm 2,2$ & $13,0 \pm 1,4$ & $8,1 \pm 1,6$ & $7,6 \pm 1,5$ & $6,0 \pm 1,5$ & $<0,01^{* *}$ \\
\hline Flexión de & Hombres & $-1,1 \pm 3,6$ & $-2,5 \pm 3,3$ & $-7,3 \pm 2,1$ & $-13,6 \pm 1,9$ & $-16,4 \pm 0,7$ & $-21,2 \pm 2,3$ & $-26,0 \pm 0,9$ & $<0,01^{* *}$ \\
\hline tronco $(\mathrm{cm})$ & Mujeres & $1,7 \pm 4,0$ & $1,5 \pm 2,1$ & $1,5 \pm 3,1$ & $-0,22 \pm 2,5$ & $-3,3 \pm 2,5$ & $-3,4 \pm 4,6$ & $-9,7 \pm 2,4$ & $<0,01^{* *}$ \\
\hline Juntar las & Hombres & $-1,54 \pm 2,7$ & $-7,0 \pm 3,0$ & $-8,1 \pm 4,0$ & $-23,0 \pm 0,8$ & $-26,1 \pm 1,6$ & $-27,3 \pm 1,3$ & $-30,2 \pm 0,7$ & $<0,01^{* *}$ \\
\hline \multirow[t]{2}{*}{ manos $(\mathrm{cm})$} & Mujeres & $-1,8 \pm 3,2$ & $-2,5 \pm 4,2$ & $-4,2 \pm 4,3$ & $-7,6 \pm 3,8$ & $-9,5 \pm 2,7$ & $-13,6 \pm 2,5$ & $-18,1 \pm 1,9$ & $<0,01^{* *}$ \\
\hline & Hombres & $5,1 \pm 0,6$ & $5,1 \pm 0,6$ & $6,0 \pm 0,3$ & $7,4 \pm 0,6$ & $9,5 \pm 0,5$ & $9,5 \pm 0,4$ & $12,6 \pm 1,4$ & $<0,01^{* *}$ \\
\hline
\end{tabular}




\begin{tabular}{llcccccccc}
$\begin{array}{l}\text { Agilidady } \\
\text { equilibrio (seg) }\end{array}$ & Mujeres & $5,8 \pm 0,5$ & $5,7 \pm 0,5$ & $6,2 \pm 0,6$ & $6,1 \pm 0,6$ & $8,9 \pm 0,4$ & $10,3 \pm 0,7$ & $12,7 \pm 0,8$ & $<0,01^{* *}$ \\
$\begin{array}{l}\text { Marcha } \\
\text { estacionaria }\end{array}$ & Hombres & $88,4 \pm 3,0$ & $87,4 \pm 1,6$ & $83,0 \pm 1,7$ & $76,2 \pm 4,0$ & $66,0 \pm 3,4$ & $55,7 \pm 2,3$ & $47,3 \pm 2,6$ & $<0,01^{* *}$ \\
(rep) & Mujeres & $80,5 \pm 5,4$ & $77,1 \pm 2,8$ & $67,3 \pm 3,1$ & $64,2 \pm 1,7$ & $54,7 \pm 1,9$ & $46,6 \pm 3,3$ & $40,1 \pm 3,5$ & $<0,01^{* *}$ \\
\hline
\end{tabular}

\section{Discusión}

La condición física funcional en los adultos mayores puede verse afectada por múltiples factores, principalmente por el envejecimiento. Por tanto, envejecer bien ha sido una preocupación constante(22). Evidencia emergente indica que la condición física es un parámetro de salud y funcionalidad en esta población.

Los cambios asociados al envejecimiento impactan significativamente la composición corporal. El IMC identificado en el presente estudio fue de $24,9 \mathrm{~kg} / \mathrm{m}^{2}$ en la población total; esto significa que tanto en hombres como mujeres la diferencia es de $3 \%$ con respecto a lo encontrado en estudios como el de Kyrdalen et al.(23), Reitlo et al.(24) y Kim et al.(25). Sin embargo, aunque el IMC como parámetro de adiposidad corporal, es un indicador ampliamente utilizado en estudios epidemiológicos para determinar el estado nutricional, sería pertinente realizar un análisis de la composición corporal desde la relación entre la masa muscular, masa grasa y osea con el propósito de estimar factores de riesgo asociados a la aparición de la sarcopenia o la caquexia(26). Estudios longitudinales desarrollados en adultos mayores establecen que a medida que aumenta la edad, la masa muscular presenta un descenso(27-29) y la masa grasa, tiende a aumentar(30). Lo que conlleva a determinar una relación clara entre la edad, un bajo nivel de condición física y parámetros de composición corporal.

Al analizar los resultados a cerca de la condición física funcional en adultos mayores institucionalizados, se encontró que tanto hombres como mujeres a partir de los 80 años se encuentran por debajo de los valores de referencia de la SFT, propuestos por Rikli y Jones en seis pruebas que evalúan los componentes de fuerza, flexibilidad, equilibrio y resistencia(5) en lo que respecta a los hombres en las pruebas que evalúan la fuerza en miembros superiores e inferiores (flexión de codo y levantarse y sentarse) se presentó una reducción del 20\%. En cuanto a la flexibilidad, determinada por medio de las pruebas de flexión de tronco y juntar las manos, se reporta una disminución del $42 \%$ y $16 \%$ respectivamente; así mismo la agilidad y equilibrio presenta una pérdida de un 19\% y un $7 \%$ en la resistencia aérobica ( 2 minutos de marcha). Ahora bien, para el caso de las mujeres, el declive de la condición física funcional se ve reflejada en la perdida de la fuerza en miembros superiores con un $23 \%$, la resistencia con un $11 \%$ y por último la agilidad y equilibrio presenta una reducción del $9 \%$.

En relación a la fuerza de miembros inferiores (levantarse y sentarse), el presente estudio estableció en mujeres de 65 a 79 años una pérdida del $8 \%$ contra el 13\% de los estudios de referencia; en el caso de los hombres en las mismas edades se observó una pérdida del $5 \%$ con respecto al $7 \%$ reportado en países como Estados Unidos (31-34). Si bien, una óptima condición física está relacionada con la salud, estudios experimentales en adultos mayores institucionalizados demuestran que una buena condición física desencadena en una buena capacidad funcional y por tanto disminuye el riesgo de caídas ${ }^{(35}$, 36).

Al contrastar los resultados de la presente investigación, con el estudio realizado en Taiwán en una población de hombres y mujeres con edad promedio de 72,6 años, $n=12.393$, se encontraron diferencias importantes en relación a la $\mathrm{CFF}^{(37)}$; el estudio refleja que, el desempeño de la condición física en hombres en la prueba de levantarse y sentarse presenta una variación porcentual del 18\%, respecto a lo reportado; en el caso de las mujeres la diferencia es del $22 \%$. En la marcha estacionaria, se registra solo una diferencia del $2 \%$ en hombres mientras que en mujeres fue del 25\%; en la fuerza de miembros inferiores en hombres la diferencia es del $24 \%$ y en mujeres del $31 \%$; finalmente, en la prueba de juntar las manos atrás de la espalda, la diferencia fue del $64 \%$ para el caso de los hombres y $65 \%$ para las mujeres.

En un estudio comparativo realizado por HerazoBeltran cuyo propósito fue estimar las diferencias en la calidad de vida, funcionalidad y condición física en adultos mayores institucionalizados, respecto a los no institucionalizados; se encontró que la institucionalización de las personas mayores está relacionada con un aumento en los porcentajes de 
inactividad física y de limitaciones en al menos una actividad básica de la vida diaria (ABVD), lo que constituye un factor negativo en la funcionalidad de la población(38).

Por otra parte Sampaio et al.(39), realizaron un estudio en una población de adultos mayores institucionalizados con un promedio de edad de 78 años. Los resultados contrastados con los de este estudio, reflejan diferencias en cuanto a la resistencia en un 15\%, obteniendo un mayor número de repeticiones; comparado con esto, la fuerza en tren inferior en el estudio referido, aparece con un desempeño del $18 \%$, caso contrario es lo que se evidencia en la fuerza de miembros inferiores, en la que la población colombiana con $21 \%$ obtiene mejores resultados; se puede inferir que las actividades que se realizan al interior de los hogares pueden favorecer la forma física de los AM.

La calidad de vida de los adultos mayores institucionalizados, se puede mejorar a partir de la intervención de la condición física, factor fundamental para los aspectos relacionados con la cognición y la capacidad funcional. Como se determinó en el presente estudio, la perdida de capacidades, habilidades y destrezas físicas, están asociadas a estilos de vida sedentarios propios de los hogares geriátricos; por tanto, el aumento de mortalidad y baja calidad de vida, está relacionada con la condición de institucionalización, deteriorando especialmente el sistema neurológico, muscular y esquelético(40).

\section{Conclusiones}

Los resultados aportados en esta investigación permiten identificar que a nivel general los adultos mayores institucionalizados que fueron evaluados, presentan características asociadas a una baja condición física funcional, respecto a los valores de referencia generados por ls SFT. Se evidencia que los componentes de la condición física funcional se ven impactados negativamente con el paso del tiempo, lo que seguramente tiene implicaciones severas en su capacidad funcional y en el desarrollo de las actividades básicas de la vida diaria; aumentando así, el riesgo de caídas y discapacidad física en el adulto mayor.

El presente estudio, puede convertirse en un referente para la formulación de programas orientados a la promoción del ejercicio físico en los hogares geriátricos, a partir de la caracterización del adulto mayor institucionalizado.

Conflicto de intereses: Los autores declaran no tener ningún conflicto de intereses.

\section{Referencias}

1. Ministerio de Protección Social. Envejecimiento Demográfico: Colombia 1951-2020 Dinámica Demográfica y Estructuras Poblacionales. Bogotá: MinSalud 2013. Disponible en https://www.minsalud.gov.co/sites/rid/Lists/BibliotecaDi gital/RIDE/DE/PS/Envejecimiento-demografico-Colombia1951-2020.pdf

2. Flórez C, Villar L, Puerta N, Berrocal L. El proceso de envejecimiento de la población en Colombia: 1985-2050. 2015. Disponible en https://www.repository.fedesarrollo.org.co/bitstream/han dle/11445/1021/Repor_Se?sequence=1

3. Kirkwood T. Why and how are we living longer?. Experimental physiology. 2017;102(9):1067-74. Disponible en

https://physoc.onlinelibrary.wiley.com/doi/full/10.1113/E P086205

4. Callisaya M, Blizzard L, Schmidt M, McGinley J, Srikanth V. Ageing and gait variability-a population-based study of older people. Age and ageing. 2010;39(2):191-7. Disponible en

https://academic.oup.com/ageing/article/39/2/191/4131 $\underline{5}$

5. Granacher U, Mühlbauer T, Bridenbaugh S, Wehrle A, Kressig R. Altersunterschiede beim Gehen unter Einfach-und Mehrfachtätigkeit. Deutsche Zeitschrift fur Sportmedizin. 2010;61(11):258. Disponible

en https://www.germanjournalsportsmedicine.com/archiv/ar chiv-2010/heft-11/altersunterschiede-beim-gehen-untereinfach-und-mehrfachtaetigkeit/

6. Magyari P, Kilpatrick \& Schoffstall J. ACSM's Resources for the Exercise Physiologist - A Practical Guide for the Health Fitness Professional. Philadelphia: Wolters Kluwer Health. 2018. Disponible

en

https://www.academia.edu/39552220/ACSMs Resources f or the Exercise_Physiologist_A_Practical_Guide_for_the_Hea lth Fitness Professional

7. Rikli R \& Jones C. Senior fitness test manual. Human Kinetics. 2001. Disponible

en https://books.google.es/books?hl=es\&lr=\&id=NXfXxOFFOV wC\&oi=fnd\&pg=PR1\&dq=Senior+fitness+test + manual +200 $1 \&$ ots=cT71G5WkiN\&sig=JXGWtV-

IQI6rfzL51dYJtPYnKsI\#v=onepage $\& \mathrm{q}=$ =Senior $\% 20$ fitness $\% 2$ 0test $\% 20$ manual $\% 202001 \& \mathrm{f}=$ false

8. Castañeda M, Gómez J, Avellaneda L, Caballero L, Delgado J. Condición física funcional y riesgo de caídas en adultos mayores. Revista Cubana de Investigaciones Biomédicas. 2019;37(3). Disponible en http://www.revibiomedica.sld.cu/index.php/ibi/article/vie $\mathrm{w} / 125 / 133$

9. Sáez M, Jiménez L, Lueso M, García A, Castaño M, López Torres Hidalgo J. Dinapenia y función musculo-esquelética en los pacientes mayores de 65 años. Revista Clínica de Medicina de Familia. 2018;11(1):8-14. Disponible en 
http://scielo.isciii.es/scielo.php?pid=S1699695X2018000100008\&script=sci arttext\&tlng=pt

10. Cortés W, Fernández F, Sanmiguel L. Sarcopenia, una patología nueva que impacta a la vejez. Revista Colombiana de Endocrinología, Diabetes \& Metabolismo. 2018;5(1):2836.

Disponible

en http://revistaendocrino.org/index.php/rcedm/article/view $\not 339$

11. Mijangos A, De la Cruz P, Alfaro L, Ribón T. Factores de riesgo de caídas e índice de masa corporal en el adulto mayor hospitalizado. Revista Cuidarte. 2019;10(1):3. Disponible en https://dialnet.unirioja.es/servlet/articulo? codigo $=695297$ 1

12. Power G, Dalton B, Rice C. Human neuromuscular structure and function in old age: a brief review. Journal of sport and health science. 2013;2(4):215-26. Disponible en http://www.academia.edu/download/47244531/Human_n euromuscular structure and functi20160714-143031r9err9.pdf

13. Rikli R, Jones C. Development and validation of criterionreferenced clinically relevant fitness standards for maintaining physical independence in later years. The Gerontologist. 2013;53(2):255-67. Disponible en https://academic.oup.com/gerontologist/article/53/2/255 $\not 560735$

14. Paterson D, Warburton D. Physical activity and functional limitations in older adults: a systematic review related to Canada's Physical Activity Guidelines. International Journal of Behavioral Nutrition and Physical Activity. 2010;7(1):38. Disponible en https://ijbnpa.biomedcentral.com/articles/10.1186/14795868-7-38?report=reader

15. García M, González E. Variables que favorecen un envejecimiento exitoso. Estudios de Psicología. 2018;39(2):215-24. Disponible en https://dialnet.unirioja.es/servlet/articulo?codigo $=670252$ 7

16. Boyaro F, Tió A. Evaluación de la condición física en adultos mayores: desafío ineludible para una sociedad que apuesta a la calidad de vida. Revista Universitaria de la educación física y el deporte. 2014(7):6-16. Disponible en https://dialnet.unirioja.es/servlet/articulo? codigo $=582640$ $\underline{4}$

17. Maillane H, Becerra G. Procesos para la prevención y reducción de la frecuencia de caídas. MinSalud. 2014. Disponible

https://www.minsalud.gov.co/sites/rid/Lists/BibliotecaDi gital/RIDE/DE/CA/prevenir-y-reducir-la-frecuencia-decaidas.pdf

18. Makizako H, Shimada H, Tsutsumimoto $\mathrm{K}$, Lee $\mathrm{S}$, Lee SC, Harada $\mathrm{K}$, et al. Age-dependent changes in physical performance and body composition in community-dwelling Japanese older adults. Journal of Cachexia, Sarcopenia and Muscle. 2017;8(4):607-14. Disponible en https://onlinelibrary.wiley.com/doi/abs/10.1002/icsm.12 197

19. Canto-Cetina T. Sarcopenia: epidemic in the twenty-first century?. Revista de Especialidades Médico-Quirúrgicas. 2016;21(4):115-6. Disponible en https://www.medigraphic.com/pdfs/quirurgicas/rmq2016/rmq164a.pdf

20. Abreus Mora JL, González Curbelo VB, Borges Clavero MÁ. Capacidad física y equilibrio en el desempeño funcional de adultos mayores. Casas de abuelos, municipio
Cienfuegos. Revista

Universidad

Sociedad. 2018;10(5):280-286. Disponible en

$\mathrm{y}$ http://scielo.sld.cu/pdf/rus/v10n5/2218-3620-rus-10-05280.pdf

21. Moral-García JE, Al Nayf Mantas MR, López-García S, Maneiro $\mathrm{R}$, Amatria M. Estado nutricional y condición física en personas mayores activas vs. Sedentarias. Revista Internacional de Medicina y Ciencias de la Actividad Física y del Deporte. 2019. Disponible en https://repositorio.uam.es/bitstream/handle/10486/6896 30/RIMCAFD_76_8.pdf?sequence=1\&isAllowed=y

22. Yerrakalva D, Mullis $\mathrm{R}$, Mant $\mathrm{J}$. The associations of "fatness,"'fitness," and physical activity with all-cause mortality in older adults: A systematic review. Obesity. 2015;23(10):1944-56. Disponible en https://onlinelibrary.wiley.com/doi/full/10.1002/oby.211 $\underline{81}$

23. Kyrdalen IL, Thingstad P, Sandvik L, Ormstad H. Associations between gait speed and well-known fall risk factors among community-dwelling older adults. Physiotherapy research international. 2019; e1743. Disponible en https://onlinelibrary.wiley.com/doi/abs/10.1002/pri.1743

24. Reitlo S, Sandbakk S, Viken H, Aspvik N, Ingebrigtsen J, Tan X, Stensvold D. Exercise patterns in older adults instructed to follow moderate- or high-intensity exercise protocol - the generation 100 study. BMC geriatrics 2018; 18(1). Disponible https://link.springer.com/article/10.1186/s12877-018$\underline{0900-6}$

25. Kim K, Lim S, Oh T, Moon J, Choi S, Lim J, et al. Longitudinal changes in muscle mass and strength, and bone mass in older adults: gender-specific associations between muscle and bone losses. The Journals of Gerontology: Series A. 2018;Series A, 73(8), 1062-1069.. Disponible en https://academic.oup.com/biomedgerontology/article/73/ $\underline{8 / 1062 / 4372285}$

26. Gomez-Cabello A, Vicente G, Vila-Maldonado S, Casajus J, Ara I. Aging and body composition: the sarcopenic obesity in Spain. Nutricion hospitalaria. 2012;27(1):22-30. Disponible en https://europepmc.org/article/med/22566301

27. Fleg J, Morrell C, Bos A, Brant L, Talbot L, Wright J, et al. Accelerated longitudinal decline of aerobic capacity in healthy older adults. Circulation. 2005;112(5):674-82. Disponible https://www ahajournals.org/doi/full/10.1161/circulation aha.105.545459

28. Rossi A, Fantin F, Di Francesco V, Guariento S, Giuliano K, Fontana G, et al. Body composition and pulmonary function in the elderly: a 7-year longitudinal study. International Journal of Obesity. 2008;32(9):1423. Disponible en https://www.nature.com/articles/ijo2008103

29. Fantin F, Francesco VD, Fontana G, Zivelonghi A, Bissoli L, Zoico E, et al. Longitudinal body composition changes in old men and women: interrelationships with worsening disability. The Journals of Gerontology Series A: Biological Sciences and Medical Sciences. 2007;62(12):1375-81. Disponible en https://academic.oup.com/biomedgerontology/article/62/ $12 / 1375 / 539390$

30. Coin A, Sergi G, Minicuci N, Giannini S, Barbiero E, Manzato E, et al. Fat-free mass and fat mass reference values by dualenergy X-ray absorptiometry (DEXA) in a 20-80 year-old Italian population. Clinical Nutrition. 2008;27(1):87-94. Disponible 
https://www.sciencedirect.com/science/article/abs/pii/S0 261561407001884

31. Guede F, Chirosa L, Fuentealba S, Vergara C, Ulloa D, Salazar $\mathrm{S}$, et al. Características antropométricas y condición física funcional de adultos mayores chilenos insertos en la comunidad. Nutrición Hospitalaria. 2017;34(6):1319-27. Disponible en http://scielo.isciii.es/scielo.php?script=sci_arttext\&pid=S02 12-16112017000900010

32. Yang M, Guo Y, Gong J, Deng M, Yang N, Yan Y. Relationships between functional fitness and cognitive impairment in Chinese community-dwelling older adults: a cross-sectional study. BMJ open. 2018;8(5):e020695. Disponible en https://bmjopen.bmj.com/content/8/5/e020695.abstract

33. Guo Y, Yang M, Yan Y, Wang L, Gong J. Sex differentials in relationships between functional fitness and cognitive performance in older adults: a canonical correlation analysis. Scientific reports. 2018;8(1):4146. Disponible en https://www.nature.com/articles/s41598-018-22475-7

34. Cho Y-H, Mohamed O, White B, Singh-Carlson S, Krishnan V. The effects of a multicomponent intervention program on clinical outcomes associated with falls in healthy older adults. Aging clinical and experimental research. 2018:1-10. Disponible https://link.springer.com/article/10.1007/s40520-0180895-z

35. Cabezas M, Mites J, Aguilar P, Hernández J, Frómeta E. Entrenamiento funcional y recreación en el adulto mayor: influencia en las capacidades y habilidades físicas. Revista Cubana de Investigaciones Biomédicas. 2019;36(4). Disponible http://www.revibiomedica.sld.cu/index.php/ibi/article/vie $\underline{w} / 22 / 21$

36. Mazo G, Silva A, Gonçalves L, Benedetti T, Claudino R, Benetti M. Aptidão física de idosos institucionalizados: um estudo interinstitucional. Conscientiae Saúde. 2011;10(3):473-9. Disponible https://www.redalyc.org/pdf/929/92920013009.pdf

37. Lee P-F, Ho C-C, Yeh D-P, Hung C-T, Chang Y-C, Liu C-C, et al. Cross-Sectional Associations of Physical Fitness Performance Level and Sleep Duration among Older Adults: Results from the National Physical Fitness Survey in Taiwan. International Journal of Environmental Research and Public Health. 2020;17(2):388. Disponible https://www.mdpi.com/1660-4601/17/2/388/htm

38. Herazo-Beltrán Y, Quintero-Cruz MV, Pinillos-Patiño Y, García-Puello F, Núñez-Bravo N, Suarez-Palacio D. Calidad de vida, funcionalidad y condición física en adultos mayores institucionalizados y no institucionalizados. Revista Latinoamericana de Hipertensión. 2017;12(5):174-81. Disponible

en http://bonga.unisimon.edu.co/bitstream/handle/20.500.12 442/2454/Calidad\%20de\%20vida $\% 2 c \% 20$ funcionalidad $\%$ 20y\%20condici\%c3\%b3n\%20f\%c3\%adsica $\% 20$ en $\% 20 \mathrm{adu}$ $\underline{\text { ltos } \% 20 \text { mayores.pdf? sequence }=1 \& \text { isAllowed }=y}$

39. Sampaio A, Marques-Aleixo I, Seabra A, Mota J, Marques E, Carvalho J. Physical fitness in institutionalized older adults with dementia: association with cognition, functional capacity and quality of life. Aging Clinical and Experimental Research. 2020:1-10. Disponible en https://link.springer.com/content/pdf/10.1007/s40520019-01445-7.pdf

40. Muñoz M, Barón M, López K, Orozco S, Naranjo D. Proceso de atención de enfermería en el fomento de actividad física de adultos mayores institucionalizados. Revista cultura del ciudadano. 2015;12(1)50-60. Disponible en http://repositorio.unilibrepereira.edu.co:8080/Viewer/ind ex.jsp?file $=123456789 / 378 / \mathrm{Art} \% 205 \% 20 \mathrm{~F} \%$ c3\%adsica.pd $\underline{f}$ 